\title{
Health-care associated infections in children after cardiac surgery in a pediatric cardiac intensive care unit (PCICU)
}

\author{
Naveed-ur-Rehman Siddiqui ${ }^{1}$, Fatima Mir ${ }^{1}$, Omair Shakil ${ }^{1}$, Muneer Amanullah ${ }^{2}$, Anwarul Haque ${ }^{1}$ \\ Department of Pediatric and Child Health ${ }^{1}$, Department of Cardiothoracic Surgery ${ }^{2}$, Aga Khan University Hospital, \\ Karachi, Pakistan
}

Key words: health-care associated infections (HAIs); cardiac intensive care unit (CICU); pediatric cardiac surgery

J Infect Dev Ctries 2011; 5(10):748-750.

(Received 30 November 2010 - Accepted 16 February 2011)

Copyright $@ 2011$ Siddiqui et al. This is an open-access article distributed under the Creative Commons Attribution License, which permits unrestricted use, distribution, and reproduction in any medium, provided the original work is properly cited.

Health-care associated infection (HAI) represents a major complication in patients undergoing cardiac surgery [1]. In the hospital setting of developing countries, where sub-optimal infection control and resource utilization may adversely affect surgical outcome, HAI rates are predictably higher (e.g 49\% in India) than those in developed countries where rates range from $5.5 \%$ to $30.8 \%[2,3,4,5]$. We report the frequency of HAIs and the associated case fatality rate among post-cardiac surgery pediatric patients admitted to the cardiac intensive care unit (CICU), at the Aga Khan University (AKU), Karachi, Pakistan between January 2008 and June 2009. Definitions of HAIs were as specified by the Centers for Disease Control and Prevention (CDC) and the National Health Care Safety Network (NHSN) Guidelines 2008 [6].

The HAI frequency in our study cohort was $7.9 \%$ (26/329). The low incidence of HAIs in our study, compared to the Indian cohort [5], may reflect strict implementation and adherence to the institutional Infection Control (IC) policy including strict visit regulations (one parent at a time), hand hygiene and an 1:1 nurse-to-patient ratio in high dependency/intensive care areas. An inverse correlation between HAI rates and nursing hours to patient day ratio had been reported in a previous cohort [2].

Table 1 elaborates demographic features, risk factors, and common sites of infection in descending order of frequency for our group of patients [4]. Though Gram-negative bacteria comprised $62.5 \%$ of all isolates $(25 / 40)$, coagulase-negative staphylococci
(CoNS) were the most prevalent pathogens overall (9/40) (Table 2).

The case-fatality rate (CFR) for pediatric postcardiac surgery HAIs was $19.2 \%(5 / 26)$ while the overall mortality rate was only $6.0 \%$ (20/329). This four-fold difference reflects the 10-fold difference reported by Grisaru-Soen et al. [4]. Though age $(<1$ month), complex congenital heart disease, higher complexity score (RACHS->3), and blood-stream infection (BSI) have all been reported as risk factors for mortality among post-cardiac surgery children with HAIs, the small size of our sample may have been responsible for not finding them significant [4].

In conclusion, although the frequency of pediatric post-cardiac surgery HAIs in our cardiac intensive care unit in Karachi, Pakistan, is lower than that reported in prior studies, case-fatality rates are comparable. Large prospective cohort studies may be required to identify population and age specific risk factors for HAI-related mortality in our hospital. 
Table 1. Characteristics of post-cardiac surgery pediatric patients who developed HAIs

\begin{tabular}{|c|c|}
\hline Variables & $\begin{array}{c}\text { Patients with HAIs } \\
(\mathrm{n}=16)\end{array}$ \\
\hline Male, n (\%) & $16(61.5)$ \\
\hline $\begin{array}{l}\text { Age, mean (range), } \mathrm{y} \\
<1 \text { month, } \mathrm{n}(\%) \\
1 \text { month-1 year, } \mathrm{n}(\%) \\
1-5 \text { years, } \mathrm{n}(\%) \\
5-19 \text { years, } \mathrm{n}(\%)\end{array}$ & $\begin{array}{c}3.86(4 \text { days - } 19 \\
\text { years) } \\
11(42.3) \\
5(19.2) \\
2(7.7) \\
8(30.8)\end{array}$ \\
\hline Weight, mean (range), kg & $10.8(2.1-40)$ \\
\hline Height, mean (range), $\mathrm{cm}$ & $77.8(42-160)$ \\
\hline $\begin{array}{c}\text { Type of CHD, n (\%) } \\
\text { Cyanotic } \\
\text { Acyanotic } \\
\text { Complex } \\
\end{array}$ & $\begin{array}{l}13(50) \\
4(15.4) \\
9(34.6) \\
\end{array}$ \\
\hline $\begin{array}{l}\text { RACHS } 1 \text { scoring, n (\%) } \\
<3 \\
>3\end{array}$ & $\begin{array}{c}20(76.9) \\
6(23.1)\end{array}$ \\
\hline $\begin{array}{l}\text { Site of infection n (\%) } \\
\text { VAP } \\
\text { BSI } \\
\text { BSI+VAP } \\
\text { SSI } \\
\text { UTI } \\
\text { BSI+VAP+UTI }\end{array}$ & $\begin{array}{l}10(38.5) \\
9(34.6) \\
3(11.5) \\
2(7.7) \\
1(3.8) \\
1(3.8)\end{array}$ \\
\hline $\begin{array}{l}\text { Sepsis related diagnosis, } \mathrm{n}(\%) \\
\text { Sepsis } \\
\text { MOD }\end{array}$ & $\begin{array}{l}22(84.6) \\
4(15.4) \\
\end{array}$ \\
\hline Pre operative antibiotics, $\mathrm{n}(\%)$ & $23(88.5)$ \\
\hline $\begin{array}{l}\text { Pre operative MV, n (\%) } \\
\text { Length of stay in PCICU, mean } \\
\text { (range), days }\end{array}$ & $\frac{3(11.5)}{10.4(3-41)}$ \\
\hline $\begin{array}{l}\text { Duration of MV, mean (range), } \\
\text { days }\end{array}$ & $8.1(1-40)$ \\
\hline $\begin{array}{l}\text { Duration of CVC, mean (range), } \\
\text { days }\end{array}$ & $10.2(3-41)$ \\
\hline $\begin{array}{l}\text { Duration of UC, mean (range), } \\
\text { days }\end{array}$ & $9.2(2-41)$ \\
\hline ACC time, mean (range), min & $103.7(40-255)$ \\
\hline CBP time, mean (range), min & $186.5(50-420)$ \\
\hline $\begin{array}{l}\text { Outcome, } \mathrm{n}(\%) \\
\text { Discharged from PCICU } \\
\text { Expired }\end{array}$ & $\begin{array}{l}21(80.8) \\
5(19.2)\end{array}$ \\
\hline
\end{tabular}

CHD: congenital heart disease, RACHS: risk associated with congenital heart surgery, VAP: ventilator associated pneumonia, BSI: bloodstream infection, SSI: surgical site infection, UTI: urinary tract infection, MOD: multi organ dysfunction, MV: mechanical ventilation, PCICU: pediatric cardiac intensive care unit, CVC: central venous catheters, UC: urinary catheter, ACC: activated cross clamp time, CBP: cardiopulmonary bypass time.
Table 2. Etiology of HAIs in post-cardiac surgery pediatric patients in Karachi, Pakistan

\begin{tabular}{|lc|}
\hline \multicolumn{1}{|c|}{ Microorganism } & Number of isolates $(\mathbf{n}=\mathbf{4 0}) \mathbf{( \% )}$ \\
\hline $\begin{array}{l}\text { Gram-negative } \\
\text { bacteria }\end{array}$ & $\mathbf{2 5}(\mathbf{6 2 . 5 )}$ \\
Acinetobacter sp. & $5(12.5)$ \\
$\begin{array}{l}\text { Pseudomonas } \\
\text { aeruginosa }\end{array}$ & $5(12.5)$ \\
Klebsiella pneumoniae & $4(10.0)$ \\
$\begin{array}{l}\text { Haemophilus } \\
\text { influenzae }\end{array}$ & $3(7.5)$ \\
$\begin{array}{l}\text { Enterobacter sp. } \\
\text { Burkholderia cepacia }\end{array}$ & $2(5.0)$ \\
Moraxella catarrhalis & $2(15.0)$ \\
$\begin{array}{l}\text { Gemella morbilium } \\
\text { Serratia liquifacies }\end{array}$ & $1(2.5)$ \\
$\begin{array}{l}\text { Escherichia coli } \\
\text { Gram-positive } \\
\text { bacteria }\end{array}$ & $1(2.5)$ \\
$\begin{array}{l}\text { Staphylococcus } \\
\text { epidermidis }\end{array}$ & $1(2.5)$ \\
$\begin{array}{l}\text { Streptococcus } \\
\text { pneumoniae }\end{array}$ & $1(2.5)$ \\
$\begin{array}{l}\text { Beta-hemolytic } \\
\text { streptococci }\end{array}$ & $12(\mathbf{3 0 . 0})$ \\
$\begin{array}{l}\text { Fungi } \\
\text { Candida albicans }\end{array}$ & $9(22.5)$ \\
Candida tropicalis & $1(2.5)$ \\
Tricosporon bacilli & $1(2.5)$ \\
\hline & $1(2.5)$ \\
\hline
\end{tabular}




\section{References}

1. Herwaldt LA, Cullen JJ, Scholz D, French P, Zimmerman MB, Pfaller MA, Wenzel RP, Perl TM (2006) A prospective study of outcomes, healthcare resource utilization, and costs associated with postoperative nosocomial infections. Infect Control Hosp Epidemiol 27: 1291-1298.

2. Archibald LK, Manning ML, Bell LM, Banerjee S, Jarvis WR (1997) Patient density, nurse-to-patient ratio and nosocomial infection risk in a pediatric cardiac intensive care unit. Pediatr Infect Dis J 16: 1045-1048.

3. Valera M, Scolfaro C, Cappello N, Gramaglia E, Grassitelli S, Abbate MT, Rizzo A, Abbruzzese P, Valori A, Longo S, Tovo PA (2001) Nosocomial infections in pediatric cardiac surgery, Italy. Infect Control Hosp Epidemiol 22: 771-775.

4. Grisaru-Soen G, Paret G, Yahav D, Boyko V, Lerner-Geva L (2009) Nosocomial infections in pediatric cardiovascular surgery patients: a 4-year survey. Pediatr Crit Care Med 10: 202-206.
5. Hasija S, Makhija N, Kiran U, Choudhary SK, Talwar S Kapil A (2008) Nosocomial infections in infants and children after cardiac surgery. Ind J Thorac Cardiovasc Surg 24: 233-239.

6. Horan TC, Andrus M, Dudeck MA (2008) CDC/NHSN surveillance definition of health care-associated infection and criteria for specific types of infections in the acute care setting. Am J Infect Control 36: 309-332.

\author{
Corresponding author \\ Naveed-ur-Rehman Siddiqui \\ Pediatric Resident \\ Department of Pediatric and Child Health \\ Aga Khan University Hospital \\ Karachi, Pakistan \\ naveed.rehman@aku.edu
}

Conflict of interests: No conflict of interests is declared. 